\title{
Kearns-Sayre Syndrome
}

National Institute of Neurological Disorders and Stroke (NINDS)

\section{Source}

National Institute of Neurological Disorders and Stroke (NINDS). Kearns-Sayre

Syndrome Information Page.

Kearns-Sayre syndrome (KSS) is a rare neuromuscular disorder with onset usually before the age of 20 years. It is the result of abnormalities in the DNA of mitochondria - small rod-like structures found in every cell of the body that produce the energy that drives cellular functions. The mitochondrial diseases correlate with specific DNA mutations that cause problems with many of the organs and tissues in the body. KSS is characterized by progressive limitation of eye movements until there is complete immobility, accompanied by eyelid droop. It is also associated with abnormal accumulation of pigmented material on the membrane lining the eyes. Additional symptoms may include mild skeletal muscle weakness, heart block (a cardiac conduction defect), short stature, hearing loss, an inability to coordinate voluntary movements (ataxia), impaired cognitive function, and diabetes. Seizures are infrequent. Several endocrine disorders can be associated with KSS. 